\title{
Serum leucine-rich alpha-2-glycoprotein-1 with fucosylated triantennary N-glycan: a novel colorectal cancer marker
}

\author{
Eiji Shinozaki ${ }^{1 \dagger}$, Kazuhiro Tanabe ${ }^{2 *}$, Takashi Akiyoshi ${ }^{3}$, Tomohiro Tsuchida ${ }^{4}$, Yuko Miyazaki ${ }^{5}$, Nozomi Kojima ${ }^{5}$, \\ Masahiro Igarashi ${ }^{4}$, Masashi Ueno ${ }^{3}$, Mitsukuni Suenaga', Nobuyuki Mizunuma', Kensei Yamaguchi', \\ Konosuke Nakayama ${ }^{6}$, Sadayo lijima ${ }^{7}$ and Toshiharu Yamaguchi ${ }^{8}$
}

\begin{abstract}
Background: Carcinoembryonic antigen (CEA) and carbohydrate antigen (CA)19-9 are used in clinical practice as tumor markers to diagnose or monitor colorectal cancer (CRC) patients, However, their specificities and sensitivities are not ideal, and novel alternatives are needed. In this study, mass spectrometry was used to search for screening markers, focusing on glycan alterations of glycoproteins in the sera of CRC patients.

Methods: Glycopeptides were prepared from serum glycoproteins separated from blood samples of 80 CRC patients and 50 healthy volunteers, and their levels were measured by liquid chromatography time-of flight mass spectrometry (LC-TOF-MS).

Results: Leucine-rich alpha-2-glycoprotein-1 with fucosylated triantennary N-glycan (LRG-FTG) was identified as CRC marker after evaluating 30,000 candidate glycopeptide peaks. The average LRG-FTG level in CRC patients $(1.25 \pm 0.973 \mathrm{U} / \mathrm{mL})$ was much higher than that in healthy volunteers $\left(0.496 \pm 0.433 \mathrm{U} / \mathrm{mL}, P<10^{-10}\right)$, and its sensitivity and specificity exceeded those of CA19-9. The combination of CEA and LRG-FTG showed a complementary effect and had better sensitivity (84\%), specificity (90\%), and AUC (0.91 by ROC analysis) than each marker alone or any other previously reported marker. LRG-FTG alone or combined with CEA also corresponded well with patient response to treatment.
\end{abstract}

Conclusions: We identified LRG-FTG as a new CRC marker, with a sensitivity and specificity exceeding CA19-9. The combination of LRG-FTG and CEA showed much higher sensitivity and specificity than each marker alone. Further validation beyond this initial exploratory cohort is warranted.

Keywords: Leucine-rich alpha-2-glycoprotein-1, Fucosylation, N-glycan, Colorectal cancer, Tumor marker

\section{Background}

The International Agency for Research on Cancer (IARC), of the World Health Organization (WHO) (http://www.irac.fr) reported that colorectal cancer $(\mathrm{CRC})$ is the fourth most common cause of cancer death. Worldwide, more than 600,000 patients die of CRC every year [1]. According to the American Cancer Society (http://www.cancer.org), the 5-year overall

\footnotetext{
* Correspondence: tanabe.kazuhiro@mp.medience.co.jp

${ }^{\dagger}$ Equal contributors

${ }^{2}$ Medical Solution Promotion Department, Medical Solution Segment, LSI

Medience Corporation, 3-30-1 Shimura, Itabashi-ku, Tokyo, Japan

Full list of author information is available at the end of the article
}

survival of Stage I CRC patients is $90 \%$, but is less than $20 \%$ in patients who are stage IV when diagnosed [2]. Detecting early stage cancer is crucial for saving patient lives. Endoscopy is the most reliable medical CRC screening method, but it has high economic and physical burdens. On the other hand, blood tests are affordable and easily performed, but accuracy of early-stage cancer detection is limited.

Tumor markers such as carcinoembryonic antigen (CEA) and carbohydrate antigen (CA)19-9 are used in clinical practice to diagnose and monitor CRC patients, but their specificities and sensitivities are unsatisfactory. CEA, first reported by Gold and Freedman in 1965 [3], 
Table 1 Participant characteristics

\begin{tabular}{llll}
\hline & chemotherapy & surgery & healthy individuals \\
\hline sex male/female & $27 / 28$ & $16 / 9$ & $25 / 25$ \\
age median(min-max) & $63(39-80)$ & $61(27-77)$ & $39(21 / 64)$ \\
stage I/II/III/V & $0 / 0 / 6 / 46$ & $2 / 11 / 12 / 0$ & - \\
pathology por/mod/well ${ }^{a}$ & $3 / 29 / 19$ & $0 / 15 / 10$ & - \\
$\mathrm{N}$ & 55 & 25 & 50 \\
\hline
\end{tabular}

apor; poorly differentiated/mod; moderately differentiated/well; well differentiated

is a member of the immunoglobulin superfamily and thought to be involved in intracellular adhesion. High serum CEA is strongly associated with malignancies, such as CRC, breast, gastric, and pancreatic cancer, and some studies have reported an association of increased pre surgery serum CEA with risk of recurrence and poor prognosis [4]. However, CEA is not specific to malignancies and is often elevated also in benign diseases, such as liver cirrhosis, gallbladder inflammation, or metabolic syndrome [5]. CA 19-9, a ligand of E-selectin that promotes binding of cancer cells to cellular endothelium, is used as a CRC or pancreatic cancer marker. Previous publications have reported cancer-related sensitivities of $50 \%$ to $90 \%$ and specificities of $54 \%$ to $98 \%$ ) [6-8], but CA19-9 is also elevated in benign diseases [9], and is not detectable in the $7 \%$ of the population who are negative for the Lewis antigen. Proteomics [10], metabolomics [11], micro-RNA [12-14], and circulating cell-free DNA $[15,16]$ have been used to identify new highly sensitive and specific candidate CRC markers, but have not been successful.

Aberrant glycosylation of serum glycoproteins is often observed in cancer patients [17-19], particularly fucosylation following activation of fucosyltransferases [20]. Increased fucosylation of the L3 isoform of $\alpha$-fetoprotein is a sensitive and specific marker of hepatocellular carcinoma [21]. Elevation of sialyl-Lewis X antigen, a tetrasaccharide carbohydrate with the sequence Neu5Ac 2 23Gal $\beta 1-4[$ Fuc $\alpha 1-3]$ GlcNAc $\beta$, in tri-/tetraantennary-Nlinked oligosaccharides has been reported in the sera of liver and lung cancer patients [22, 23]. Although alteration of sugar chains shows promise as a cancer marker, difficulties in the analysis of the wide variety of sugar chain structures have hampered this approach.

Recent improvements of mass spectrometry in the sensitivity, resolution, and ability to rapidly analyze a large number, have revolutionized the screening of markers, specifically glycoproteins. In this study, we aimed to identify new glycoproteins as CRC markers. Our strategy was to use mass spectroscopy and liquid chromatography to analyze total glycoproteins in the sera of CRC patients and then compare candidate biomarker levels in patients with those in healthy volunteers. The advantages of this strategy are the ability to assess not only alterations of sugar chains but also those of the "host" proteins, and the ability to screen thousands (approximately 10,000 to 100,000) of glycopeptide candidates. Although screening of glycopeptide markers has been described for some specific proteins, such as haptoglobins $[24,25]$, screening of total serum glycoproteins has not been reported. We tried to find new CRC markers with potential as alternatives or complementarity to currently available markers.

\section{Methods \\ Collection of blood samples and clinical data}

We enrolled patients at the Cancer Institute Hospital of the Japanese Foundation for Cancer Research (Tokyo, Japan) with histologically confirmed adenocarcinoma of the colon or rectum. Patients with impaired renal and liver function were excluded. After obtaining informed consent from eligible patients, we assayed $2-\mathrm{mL}$ of sera that remained after performing routine laboratory tests before surgery or chemotherapy, and at 1, 3, and 6 months after anticancer treatment. Clinical data were retrieved from patient medical records, and CT images were evaluated for antitumor effect by Response Evaluation Criteria in Solid Tumors (RECIST) version 1.1. Patient characteristics including sex, age, stage, tumor histology, and treatment were collected. We obtained sera of healthy volunteers from SOIKEN (Osaka Japan) with informed consent. All healthy volunteers received medical examinations prior to blood sampling, and those whose results exceeded normal criteria in any test were not enrolled in the study. CA19-9 and CEA were analyzed by chemiluminescent immunoassay method in the Cancer Institute Hospital. This study was approved by the Institutional Review Board (No. 2011-1025) of Cancer Institute Hospital of Japanese Foundation for Cancer Research, and was conducted following the Ethical Guidelines of Ministry of Health, Labor and Welfare in Japan. Written informed consent was obtained from all patients before participation.

\section{Serum preparation}

Sera were prepared by previously described methods $[26,27]$. Briefly, $400 \mu \mathrm{L}$ of cold acetone containing 


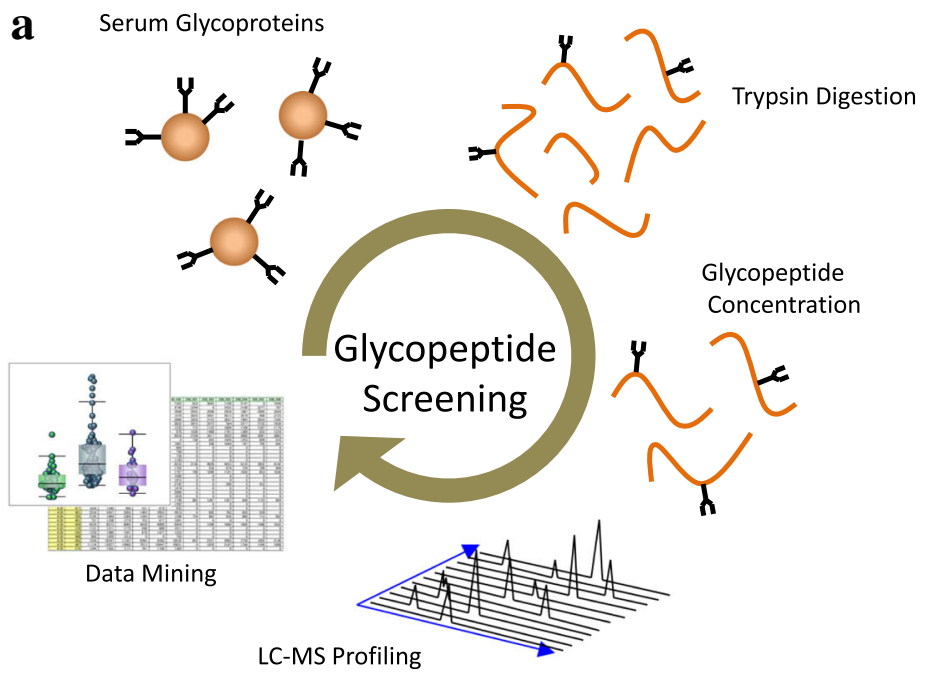

b

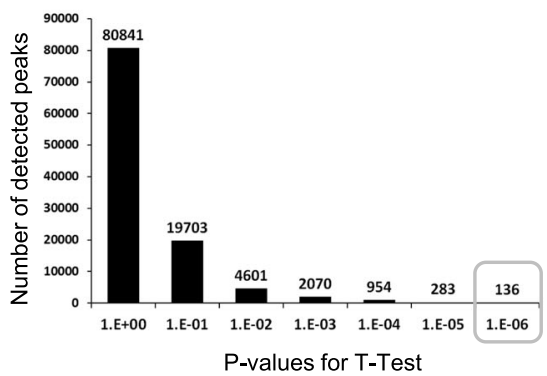

d

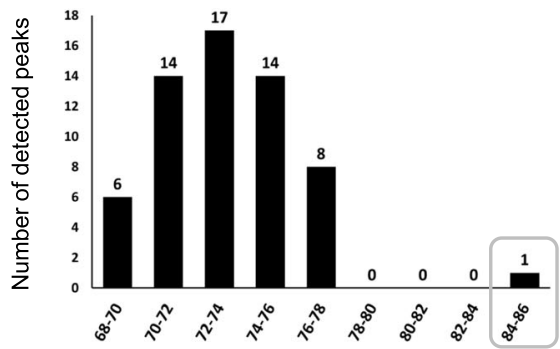

C

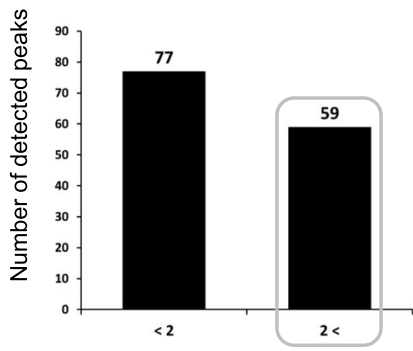

Fig. 1 Screening of CRC cancer markers. a All isolated serum glycoproteins were digested by trypsin to form peptides, and the glycopeptides were enriched by ultrafiltration and AAL lectin chromatography. Then they were analyzed by LC-TOF-MS. Glycopeptide peak positions (m/z and elution time) and peak intensities (peak areas) were calculated by software developed in our laboratory. The glycopeptide peaks obtained for all serum samples were then aligned and included in a single table, i.e., a peak list. Finally, CRC markers were screened by t-test statistics, mean-fold change analysis, and ROC analysis. CRC markers were extracted with t-test values $P<10^{-6}$, (b), mean-fold change analysis with ratios $>2$ (c), and ROC analysis with AUCs $>0.80$ (d). The values of the marker were normalized against levels of healthy controls (HEA219)

$10 \%$ trichloroacetic acid (Wako Pure Chemical Industries, Ltd., Osaka, Japan), and $50 \mu \mathrm{g}$ of fetal calf fetuin (Sigma, St. Louis, MO, USA) as an internal standard, were added to $100 \mu \mathrm{L}$ of patient sera, and mixed at $-20{ }^{\circ} \mathrm{C}$ for $90 \mathrm{~min}$ to remove serum albumin. After the mixture was centrifuged at $13000 \mathrm{~g}$ at $4{ }^{\circ} \mathrm{C}$ for $20 \mathrm{~min}$, the supernatants were removed and the precipitates were washed with $400 \mu \mathrm{L}$ of cold acetone to remove excess trichloroacetic acid. After
$13,000 \mathrm{~g}$ centrifugation, the precipitates were mixed with $1 \mathrm{~mL}$ denaturing solution, $40 \%(w / v)$ of urea (Wako Pure Chemical Industries), $0.5 \mathrm{M}$ Tris- $\mathrm{HCl}$ buffer (pH 8.5), 5 mM EDTA, 40 mM Tris (2-carboxyethyl) phosphine hydrochloride (Sigma). Then $100 \mu \mathrm{L}$ of $1 \mathrm{M}$ 2-iodoacetamide (Wako Pure Chemical Industries) solution was added to denatured proteins and reacted at $37{ }^{\circ} \mathrm{C}$ for $1 \mathrm{~h}$ to protect the free thiol residues. The solutions were transferred into 


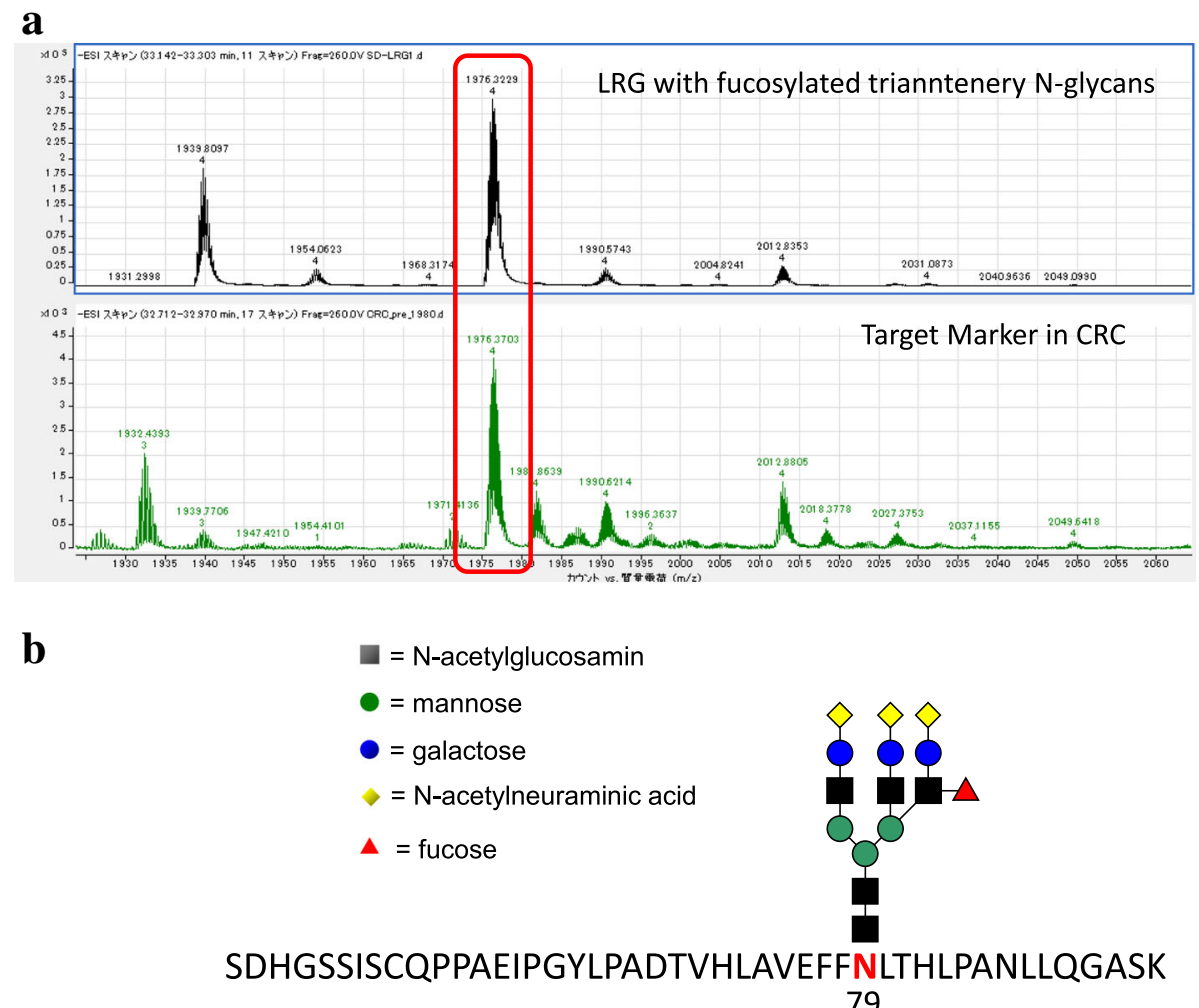

Fig. 2 Identification of CRC marker glycopeptides. The mass spectrum of the CRC marker was incorporated into a database that included the $\mathrm{m} / \mathrm{z}$ and retention times of glycopeptides generated from standard serum proteins by trypsin digestion. (a) mass spectrum of standard LRG glycopeptides (upper), and mass spectrum of target marker glycopeptides detected in CRC patients (lower), and (b) the proposed structure of the CRC marker

Amicon Ultra $30 \mathrm{~K}$ 4-mL centrifugal filtration tubes (Millipore Corp., MA, USA) and centrifuged at $3000 \mathrm{~g}$ for $30 \mathrm{~min}$ to remove denaturing reagents. The proteins trapped on the filters were washed with $2 \mathrm{~mL}$ of $0.1 \mathrm{M}$ Tris- $\mathrm{HCl}$ buffer ( $\mathrm{pH} 8.5$ ), and then they were centrifuged at $3000 \mathrm{~g}$ for $40 \mathrm{~min}$. Next, $1 \mathrm{~mL}$ of $0.1 \mathrm{M}$ Tris- $\mathrm{HCl}$ buffer $(\mathrm{pH} 8.5), 100 \mu \mathrm{L}$ of 0 . $1 \mu \mathrm{g} / \mu \mathrm{L}$ trypsin solution, and $100 \mu \mathrm{L}$ of $0.1 \mu \mathrm{g} / \mu \mathrm{L}$ lysyl endopeptidase (Wako Pure Chemical Industries) solution were added into the Amicon tubes and the proteins were digested at $37{ }^{\circ} \mathrm{C}$ for $16 \mathrm{~h}$. The solution was centrifuged at $3000 \mathrm{~g}$ for $10 \mathrm{~min}$, and the supernatant containing peptides was transferred into an Amicon Ultra $10 \mathrm{~K}$, 4-mL tube (Millipore Corp.), then it was centrifuged at $3000 \mathrm{~g}$ for $10 \mathrm{~min}$. Most peptides with sugar chains were trapped on the $10 \mathrm{~K}$ ultra-filter, whereas most nonglycosylated peptides were removed by the filtration. The glycopeptides trapped on the filter were washed with $2 \mathrm{~mL}$ of a solution containing 10\% acetonitrile and $90 \% 10 \mathrm{mM}$ ammonium acetate, then they were transferred into a $1.5-\mathrm{mL}$ microtube to be dried up by vacuum centrifuging.

Two mg of Aleuria aurantia lectin (AAL, Vector, Burlingame, CA, USA) hold on one $\mathrm{mL}$ of agarose gel was placed in an empty one-mL column (Agilent, CA, USA) and it was washed with $30 \mathrm{~mL}$ of $0.1 \mathrm{M}$ Tris- $\mathrm{HCl}$ buffer ( $\mathrm{pH}$ 7.4). Enriched glycopeptide fraction prepared from $40 \mu \mathrm{L}$ of serum, was dissolved in $200 \mu \mathrm{L}$ of water and loaded onto the lectin column. After it was washed with $15 \mathrm{~mL}$ of $10 \mathrm{mM}$ Tris- $\mathrm{HCl}$ buffer ( $\mathrm{pH} 7.4$ ), only fucosylated glycopeptides were eluted with $15 \mathrm{~mL}$ of $100 \mathrm{mM}$ fucose solution. The eluted solution was transferred into an Amicon Ultra $3 \mathrm{~K}$ tubes $(15 \mathrm{~mL})$ and centrifuged at $4000 \mathrm{~g}$ for $90 \mathrm{~min}$ to remove excess fucose. The fucosylated glycopeptides remaining on the filter were washed with further $10 \mathrm{~mL}$ of water and they were transferred into LC vial tubes to be analyzed by mass spectroscopy (LC-MS).

\section{Screening of CRC markers by LC-MS}

LC-MS data sets were acquired with a liquid chromatograph (Agilent HP1200, Agilent Technologies, Palo Alto, CA), an electrospray ionization quadrupole and time of flight (Q-TOF) mass spectrometer (Agilent 6520, Agileent Technologies). An Inertsil C18 column $(2 \mu \mathrm{m}, 100 \mathrm{~mm} \times 1.5 \mathrm{~mm}$ ID, GL Science, Tokyo, Japan) was used for high-performance liquid chromatography (HPLC). Solvent A was $0.1 \%$ formic acid 

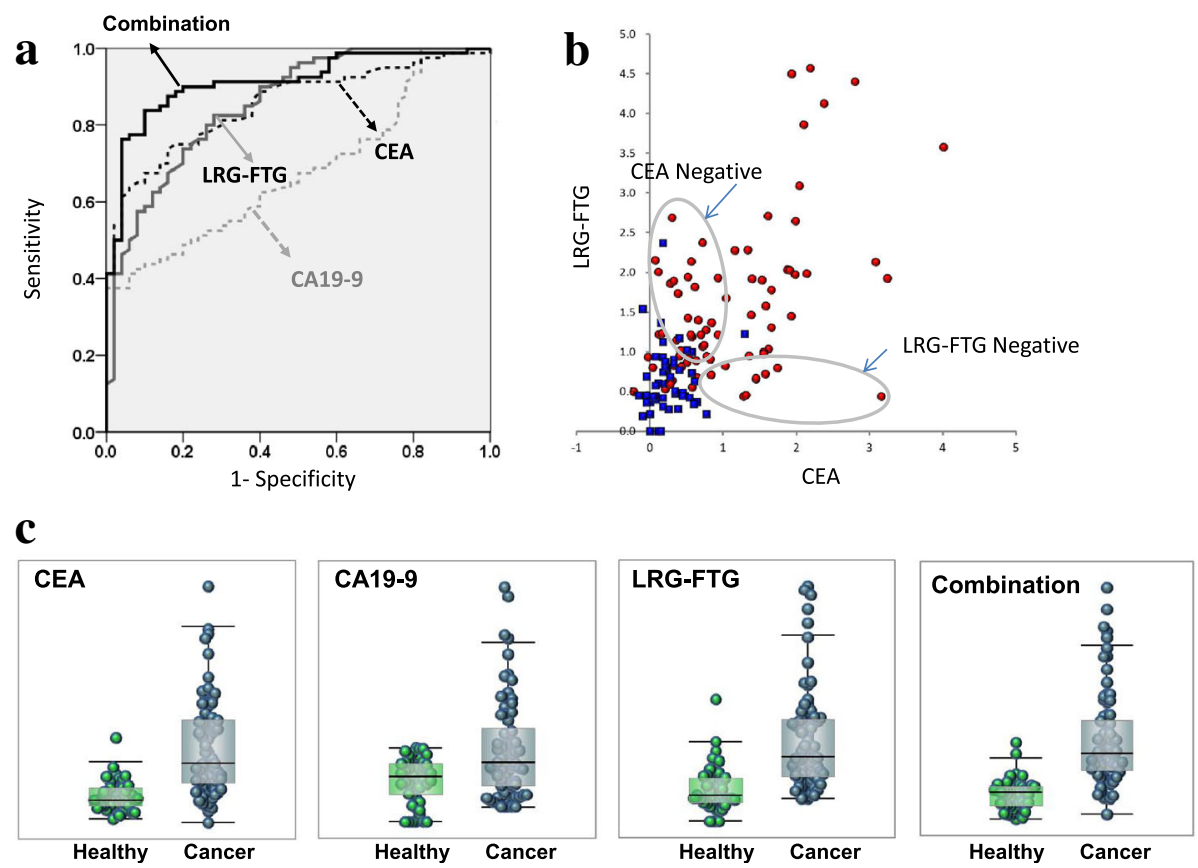

Fig. 3 Diagnostic performance of LRG-FTG, and currently used CRC markers (CEA and CA19-9). a ROC curves comparing 80 CRC patients with 50 healthy volunteers for CEA, CA19-9, LRG-FTG, and the combination. b Box and whisker plots of CEA, CA19-9, LRG-FTG and the combination. $\mathbf{c}$ Scatter plots of CEA (Log10) and LRG-FTG. Red and blue circles represent CRC and healthy volunteers respectively. Combination values are calculated as: Combination factor $=\log 10(C E A) \times 0.8+(L R G-F T G) \times 0.6$

aqueous solution and solvent B was $0.1 \%$ of formic acid, 9 . $9 \%$ water and $90 \%$ acetonitrile. Glycopeptides were eluted at a flow rate of $0.1 \mathrm{~mL} / \mathrm{min}$ at $40{ }^{\circ} \mathrm{C}$ with a linear gradient of $10 \%-56 \%$ of Solvent B over $40 \mathrm{~min}$ and a further $10 \mathrm{~min}$ hold at $100 \%$ of Solvent B. The mass spectrometer was operated in the negative mode. The capillary voltage was set at $4000 \mathrm{~V}$. Nebulizing gas pressure was $30 \mathrm{psi}$, and the dry gas flow was $8 \mathrm{~L} / \mathrm{min}$ at $350{ }^{\circ} \mathrm{C}$.

\section{Screening of CRC markers}

The mass spectrometry data was analyzed using Marker Analysis, the software developed in our laboratory [27]. After all peak positions (retention time and $\mathrm{m} / \mathrm{z}$ ) and intensities (peak areas) were calculated, the peaks of all samples from patients and healthy volunteers were aligned, to generate a peak list. The errors generated in preparation and the LC-MS analysis step, were corrected using the internal standard peptides derived from fetal calf fetuin, and were normalized against glycopeptides obtained from a healthy person (HEA219). Marker screening analysis was performed by t-test statistics, mean-fold change analysis, and receiver operating characteristic (ROC) curve analysis using Marker Analysis and SPSS 17.0 (SPSS, Chicago, IL). Eighty CRC patients and 50 healthy volunteer control persons were compared. ROC curve analysis was used to determine optimum cutoff values of candidate markers and maximize sensitivity and specificity.

\section{Identification of CRC markers}

We previously established a database of retention times and $\mathrm{m} / \mathrm{z}$ of glycopeptides generated from trypsin digestion of standard proteins. Standard human serum glycoproteins, such as alpha-1-acid glycoprotein, alpha-1-antitrypsin, clusterin, haptoglobin, kininogen, leucine rich glycoprotein, alpha-2-macrogloblin, ceruloplasmin, transferrin, immunoglobulin, and complement C3, were obtained from Sigma (St. Louis, MO, USA). After these proteins were digested by trypsin, and the glycopeptides were analyzed by LC-MS, they were further treated with PNGase F (New England Biolabs, Ipswich, MA, USA) to remove N-glycans. The sugar-free peptides were analyzed by tandem mass spectroscopy (MS/MS), and the data were analyzed by Mascot (Matrix Science, version 2.3.02), using the UniProt/SwissProtein sequence database (October 2015, 20,266 total sequences). Database search parameters were restricted to one missed tryptic cleavage site, a precursor ion mass tolerance of $1.2 \mathrm{Da}$, a fragment ion mass tolerance of $0.6 \mathrm{Da}$ and a $P$-value of $<0.05$. The sugar chain compositions were suggested by the delta of molecular weight between preand post- PNGase F glycopeptide digestion. The analytical data (retention time and $\mathrm{m} / \mathrm{z}$ of each peptide) was registered with the protein names, the sugar chain binding sites, and the proposed sugar chain structures. All 
Table 2 Treatment outcomes 6 months following surgery or chemotherapy

\begin{tabular}{ll}
\hline Chemotherapy & \\
RECIST CR/ PR/ SD/PD a & $0 / 10 / 19 / 14$ \\
Surgery & \\
RO/ others & $25 / 0$ \\
Recurrence yes/ no & $0 / 25$ \\
\hline
\end{tabular}

${ }^{a} \mathrm{CR}$, complete response; $\mathrm{PR}$, partial response; $\mathrm{SD}$, stable disease; $\mathrm{PD}$, progressive disease

glycopeptide peaks detected in CRC and healthy volunteer sera were imported into the database and assigned protein and sugar chain structures.

\section{Results}

Screening and identification of new CRC biomarker

Eighty CRC patients and 50 healthy volunteers were enrolled (Table 1), and follow up samples were obtained from 68 of the patients. A new CRC marker was identified after screening over 30,000 glycopeptide peaks following the schema shown in Fig. 1a. First, 136 CRC peaks (0.13\%) with a t-test $P<10^{-6}$ compared with healthy volunteers (Fig. 1b) were selected. Of these, 59 peaks $(0.026 \%)$ with a mean-fold change $>2$ were selected (Fig. 1c), and finally one glycopeptide with an area under the curve (AUC) by
ROC curve analysis $>0.80$ was isolated as the best CRC marker candidate (Fig. 1d). The elution time (33 $\mathrm{min}$ ) and $\mathrm{m} / \mathrm{z}$ (1975.37, z4) of this marker was just coincident with leucine-rich alpha-2-glycoprotein-1, whose Asn79 was modified with fucosylated triantennary N-glycan (LRGFTG, Fig. 2a, b). The sugar chain structure was proposed as A3G3S3F from the molecular weight delta of pre- and post-PNGase digestion. The sugar chain composition was proposed as six hexoses, five hexNAcs, three $\mathrm{N}$-acetyl neuraminic acids, and one fucose, Fig. 2b). The average LRGFTG concentration in CRC patients was $1.25 \pm 0.973 \mathrm{U} / \mathrm{mL}$ and $0.496 \pm 0.433 \mathrm{U} / \mathrm{mL}$ in healthy volunteers $\left(\mathrm{P}<10^{-10}\right.$ by $\mathrm{t}$-test). LRG with biantennary glycan did not change between CRC patients and healthy individuals (Additional file 1: Figure S1), which meant that the sugar chain alteration in LRG was key to cancer diagnosis.

\section{Comparison of LRG-FTG with current markers, CEA and CA19-9}

Figure 3 shows box and whisker plots and ROC curves that compare the AUCs of LRG-FTG, CEA and CA199 in patients and healthy volunteers. The AUC of LRGFTG (0.86) was significantly greater than that of CA199 (0.68), but was almost equal to that of CEA (0.85, Fig. $3 \mathrm{a})$. The sensitivity of LRG-FTG was $80 \%$, and the specificity was $74 \%$, when the cutoff value was $0.82 \mathrm{U} / \mathrm{mL}$.

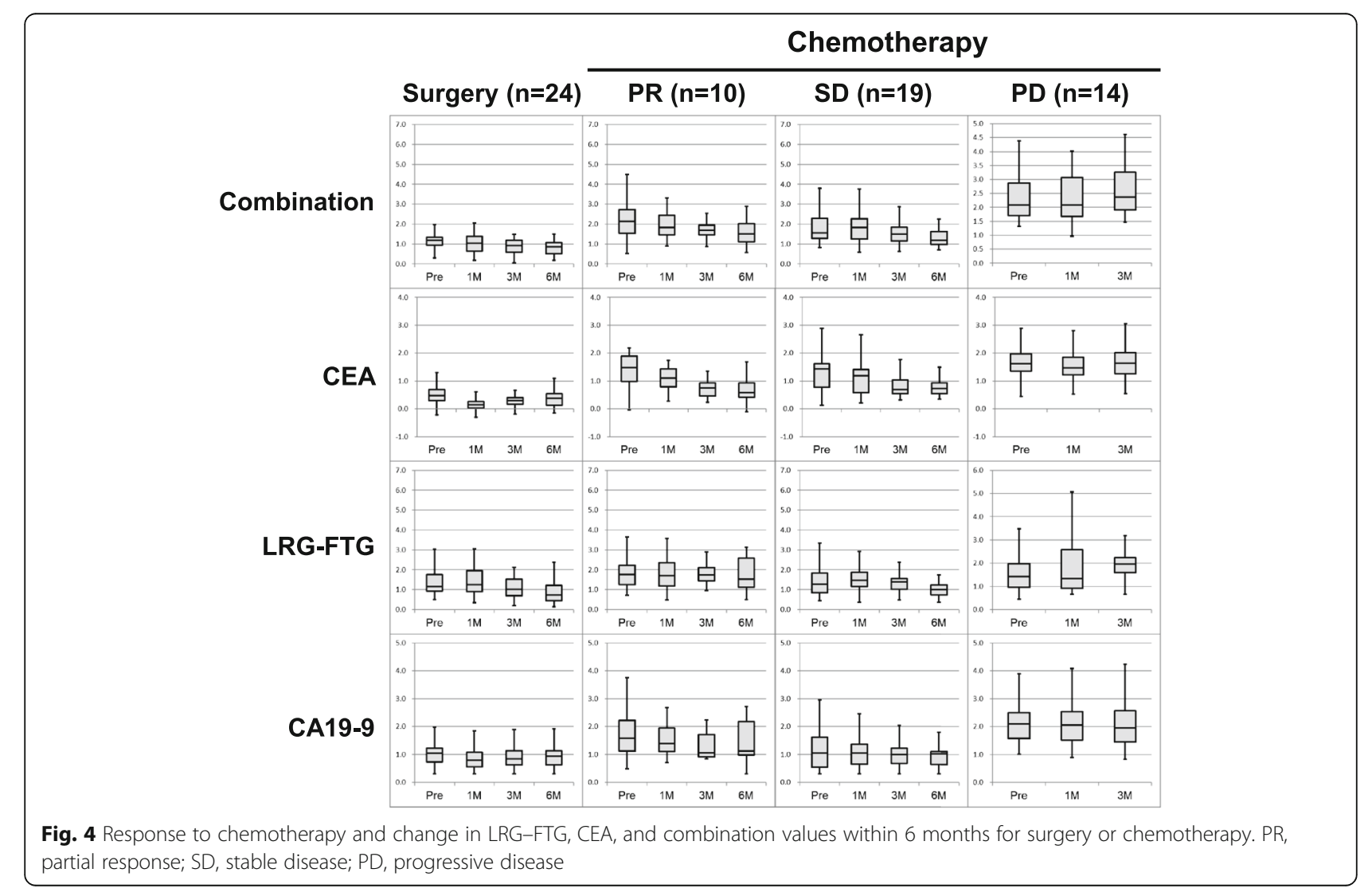


The cutoff value was the one that resulted in the highest sensitivity and specificity.

There was a weak correlation between LRG-FTG and CEA $(r=0.61)$, however their relation was complementary, as 24 of $33(73 \%)$ CEA-negative $(<5 \mathrm{ng} / \mathrm{mL})$ patients would be considered positive by LRG-FTG (Fig. 3b). Furthermore, a combination factor, calculated by the next formula, resulted in a much higher AUC (0.91) compared with CEA or LRG-FTG alone (Fig. 3a, c). The coefficients of this formula were optimized by EXCEL Solver program (Additional file 2).

Combination factor $=\log 10(C E A) \times 0.8+(L R G-F T G)$ $\times 0.6$.

The sensitivity and specificity of the combination became $84 \%$ and $90 \%$ respectively when the cutoff was $0.92 \mathrm{U} / \mathrm{mL}$.

\section{Multiple glycopeptide combination screening}

Orthogonal partial least squares discriminant analysis (OPLS-DA, SIMCA, Umetrics, Sweden) using 136 glycopeptides screened by t-test was performed with an aim to separate CRC patients from healthy individuals. The Q2 score, a predictive value using cross validation technique, showed reliable level (0.37) and the ROC-AUC using t1 score indicated high level (0.92, Additional file 3: Figure S2).

\section{Correlation of LRG-FTG and short-term treatment outcome}

To investigate the correlation of LRG-FTG and outcomes of surgery or chemotherapy, the 68 patients who were available for follow-up were monitored for 6 months after treatment. Prior to analysis, the patients were assigned to four groups. The first included 25 patients with complete surgical resection and no metastases or recurrences within the 6-month monitoring period. The other three groups were defined by the effectiveness of chemotherapy as partial response (PR, $n=10)$, stable disease (SD, $n=19$ ), and progressive disease (PD, $n=14$, Table 2). Changes in the LRG-FTG, CEA, and combined values observed in the four groups are shown in Fig. 4. Compared three months after treatment to pre-treatment of $\mathrm{PD}$ patients, LRG-FTG showed good correlation with treatment outcome (elevated in 11/14 patients), whereas CEA did not correspond well with the outcome (elevated in 6/14 patients, Additional file 4). The combined LRG-FTG and CEA value was the most closely associated with patient outcome. It continuously increased in patients with PD and decreased in those with a PR. In the surgery group, the level gradually decreased during follow up and was below the cutoff of $0.92 \mathrm{U} / \mathrm{mL}$ at 6 months.

\section{Discussion}

In this study, we identified LRG-FTG as a new CRC marker, with a sensitivity and specificity exceeding CA19-9. Its sensitivity and specificity were almost equal to CEA, but when LRG-FTG and CEA were combined, the effect was complementary, achieving a sensitivity of $84 \%$, specificity of $90 \%$, and AUC of 0.91 by ROC analysis, all of which were higher than each marker alone.

LRG (leucine-rich alpha-2-glycoprotein-1) is a protein involved in an the acute-phase response to bacterial or viral infection [28], or the initiation of granulocyte differentiation. Elevation of serum LRG has been observed in various disease states, including toxic-shock syndrome [29], inflammation in cystic fibrosis [30], ovarian cancer [31], pancreatic cancer [32-34]. Wang et al. reported that LRG promoted angiogenesis in cancer progression by binding to the transforming growth factor (TGF) $-\beta$ accessory receptor [35], and was considered as a potential cancer marker. In this patient series, as LRG-FTG was only weakly correlated $(r=0.47)$ with an inflammatory marker, CRP (Additional file 5: Figure S3), the relationship might be incompletely dependent.

There have been some reports of increased serum LRG in cancer patients, but few have mentioned LRG sugar-chain alterations. Aberrant glycosylation has been observed in serum glycoproteins of cancer patients. Increased $\alpha 1-3 / 4$ fucosylation (Lewis $\mathrm{X} / \mathrm{A}$ ) of highly branched $\mathrm{N}$-glycans has been identified in liver and lung cancer [22]. There has also been a report of an association of aberrant glycosylation with promotion of cancer metastasis [36]. LRG-FTG might thus be involved in not only cancer development, but also cancer metastasis.

Sandanayake et al. proposed that the combination of serum LRG, CA19-9, and interleukin-6 was an effective marker, able to distinguish biliary tract cancer from benign biliary disease, which is difficult using CA19-9 alone [37]. This indicates that using multiple markers that combine several characteristic proteins or sugar chains, rather than single markers, could improve the accuracy of diagnosis. The US Food and Drug Association has recently approved a panel of biomarkers to aid in the diagnosis of ovarian cancer prior to surgery [38], the combination of several biomarkers would be used for various cancers. To develop LRG-FTG marker as a clinical test, two approaches can be considered. One is to develop a lectin-antibody sandwich assay, and the other is to simplify LC-MS method. A lectin-antibody sandwich assay is superior in terms of test cost, however, the low specificity of lectins (recognition of a target sugar chain) remains challenging. On the other hand, LC-MS has prevented its clinical use due to its lowthroughput, however, ultra-high performance liquid chromatography technique dramatically improved its performance, and its clinical use is now insight. 


\section{Conclusion}

Serum LRG-FTG was significantly elevated in CRC patients compared with healthy volunteers, and its accuracy as a CRC tumor marker was equal to that of CEA. Moreover, combined with CEA, it had an excellent profile. LRG-FTG values corresponded well with treatment outcome for the patients with PD. LRG-FTG is expected to be an alternative marker for diagnosis of CRC, however further validation beyond this initial exploratory cohort is warranted.

\section{Additional files}

Additional file 1: Figure S1. LRG with biantennary glycans of CRC patients and healthy individuals. (a) A box plot of LRG with biantennary glycans between CRC patients and healthy individuals, (b) The target glycopeptide structure of LRG with biantennary glycans. (PPTX 90 kb)

Additional file 2: Coefficient Optimization using EXCEL Solver. Process of coefficient optimization of combination assay. (XLSX 18 kb)

Additional file 3: Figure S2. OPLS-DA analysis using 136 glycopeptides extracted by t-test. (a) A score plot for 80 CRC patients and 50 healthy in dividuals. (b) R2 and Q2 plot for two components. (C) ROC analysis for OPLS-DA t1 score. (PPTX $140 \mathrm{~kb}$ )

Additional file 4: Raw Data. Primary data for analysis. (XLSX 39 kb)

Additional file 5: Figure S3. Relationship of serum LRG-FTG and Creactive protein in $80 \mathrm{CRC}$ patients. Scatter plots showing the relation LRG-FTG and CRP. (PPTX $68 \mathrm{~kb}$ )

\section{Abbreviations}

CEA: Carcinoembriyonic antigen; CRC: Colorectal cancer; LRG_FTG: Leucinerich alpha-2-glycoprotein-1 with fucosylated triantennary N-glycan

\section{Acknowledgements}

We would like to express our great appreciation to Keiko Nakano for analyzing glycopeptides in patient's sera,

\section{Funding}

All sources of funding for the research, including design of the study and collection, analysis, and interpretation of data and writing the manuscript were provided by Mitsubishi Chemical Group Science and Technology Research Center.

\section{Availability of data and materials}

The datasets generated and/or analyzed during the current study are not publicly available because the intellectual property of this study belongs to Mitsubishi Chemical Group Science and Technology Research Center and LSI Medience Corporation, and some files includes highly confidential data that has not been approved as patents. But they can be disclosed from the corresponding author on reasonable request, and the data that support the findings of this study are publicly available as supplemental data on this journal's web site.

\section{Authors' contributions}

All authors read and approved the final manuscript. ES, a principal investigator, managed this project, and evaluated the marker's clinical significances. KT established the method of glycopeptide analysis using mass spectrometry, and built the data mining program. TA designed this study, acquired the data, and evaluated the marker's clinical significances. TT designed this study, acquired the data, and evaluated the marker's clinical significances. YM analyzed glycopeptides of CRC patients and contributed to finding new biomarkers. NK analyzed glycopeptides of CRC patients and contributed to finding new biomarkers. MI designed this study, acquired the data, and evaluated the marker's clinical significances. MU designed this study, acquired the data, and evaluated the marker's clinical significances. MS designed this study, acquired the data, and evaluated the marker's clinical significances. NM designed this study, acquired the data, and evaluated the marker's clinical significances. KY designed this study, acquired the data, and evaluated the marker's clinical significances. KN designed this study, acquired the data, and evaluated the marker's clinical significances. SI contributed to organize this study and managed the project. TY contributed to organize this project, and evaluated the marker's clinical significances.

\section{Ethics approval and consent to participate}

This study was approved by the Institutional Review Board (No. 2011-1025) of Cancer Institute Hospital of Japanese Foundation for Cancer Research, and was conducted following the Ethical Guidelines of Ministry of Health, Labor and Welfare in Japan. Written informed consent was obtained from all patients before participation.

\section{Consent for publication}

Not applicable.

\section{Competing interests}

The authors declare that they have no competing interests. Kazuhiro Tanabe and Sadayo lijima are working for LSI Medience Corporation, and Yuko Miyazaki and Nozomi Kojima are woking for Mitsubishi Chemical Group Science and Technology Research Center.

\section{Publisher's Note}

Springer Nature remains neutral with regard to jurisdictional claims in published maps and institutional affiliations.

\section{Author details}

${ }^{1}$ Department of Gastrointestinal Oncology, Cancer Institute Hospital of Japanese Foundation for Cancer Research, Tokyo, Japan. ${ }^{2}$ Medical Solution Promotion Department, Medical Solution Segment, LSI Medience Corporation, 3-30-1 Shimura, Itabashi-ku, Tokyo, Japan. ${ }^{3}$ Department of Gastrointestinal Surgery, Cancer Institute Hospital of Japanese Foundation for Cancer Research, Tokyo, Japan. ${ }^{4}$ Department of Gastroenterology, Cancer Institute Hospital of Japanese Foundation for Cancer Research, Tokyo, Japan. ${ }^{5}$ Biotechnology Laboratory Mitsubishi Chemical Group Science and Technology Research Center, Inc, Tokyo, Japan. ${ }^{6}$ Department of Internal Medicine, Cancer Institute Hospital of Japanese Foundation for Cancer Research, Tokyo, Japan. 'International Sales Department, LSI Medience Corporation, Tokyo, Japan. ${ }^{8}$ Cancer Institute Hospital of Japanese Foundation for Cancer Research, Tokyo, Japan.

Received: 8 August 2017 Accepted: 20 March 2018

Published online: 11 April 2018

References

1. Siegel RL, Miller KD, Fedewa SA, Ahnen DJ, Meester RGS, Barzi A, Jemal A Colorectal cancer statistics, 2017. CA Cancer J Clin. 2017;67(3):177-93.

2. Brenner H, Kloor M, Pox CP. Colorectal cancer. Lancet (London, England). 2014;383(9927):1490-502.

3. Gold P, Freedman SO. Specific carcinoembryonic antigens of the human digestive system. J Exp Med. 1965;122(3):467-81.

4. Lee JH, Kim SH, Jang HS, Chung HJ, Oh ST, Lee DS, Kim JG. Preoperative elevation of carcinoembryonic antigen predicts poor tumor response and frequent distant recurrence for patients with rectal cancer who receive preoperative chemoradiotherapy and total mesorectal excision: a multiinstitutional analysis in an Asian population. Int J Color Dis. 2013;28(4):511-7.

5. Kim KN, Joo NS, Je SY, Kim KM, Kim BT, Park SB, Cho DY, Park RW, Lee DJ. Carcinoembryonic antigen level can be overestimated in metabolic syndrome. J Korean Med Sci. 2011;26(6):759-64.

6. Chalasani N, Baluyut A, Ismail A, Zaman A, Sood G, Ghalib R, McCashland TM, Reddy KR, Zervos X, Anbari MA, et al. Cholangiocarcinoma in patients with primary sclerosing cholangitis: a multicenter case-control study. Hepatology (Baltimore, Md). 2000;31(1):7-11.

7. Levy C, Lymp J, Angulo P, Gores GJ, Larusso N, Lindor KD. The value of serum CA 19-9 in predicting cholangiocarcinomas in patients with primary sclerosing cholangitis. Dig Dis Sci. 2005;50(9):1734-40.

8. Patel AH, Harnois DM, Klee GG, LaRusso NF, Gores GJ. The utility of CA 19-9 in the diagnoses of cholangiocarcinoma in patients without primary sclerosing cholangitis. Am J Gastroenterol. 2000;95(1):204-7. 
9. Tong Y, Song Z, Zhu W. Study of an elevated carbohydrate antigen 19-9 concentration in a large health check-up cohort in China. CCLM / FESCC. 2013;51(7):1459-66.

10. Snoeren N, Emmink BL, Koerkamp MJ, van Hooff SR, Goos JA, van Houdt WJ, de Wit M, Prins AM, Piersma SR, Pham TV, et al. Maspin is a marker for early recurrence in primary stage III and IV colorectal cancer. $\mathrm{Br} J$ Cancer. 2013;109(6):1636-47.

11. Zhu J, Djukovic D, Deng L, Gu H, Himmati F, Chiorean EG, Raftery D. Colorectal cancer detection using targeted serum metabolic profiling. J Proteome Res. 2014;13(9):4120-30.

12. Zheng G, Du L, Yang X, Zhang X, Wang L, Yang Y, Li J, Wang C. Serum microRNA panel as biomarkers for early diagnosis of colorectal adenocarcinoma. Br J Cancer. 2014;111(10):1985-92.

13. Yau TO, Wu CW, Dong Y, Tang CM, Ng SS, Chan FK, Sung JJ, Yu J. microRNA-221 and microRNA-18a identification in stool as potential biomarkers for the non-invasive diagnosis of colorectal carcinoma. $\mathrm{Br}$ J Cancer. 2014;111(9):1765-71.

14. Zanutto S, Pizzamiglio S, Ghilotti M, Bertan C, Ravagnani F, Perrone F, Leo E, Pilotti S, Verderio P, Gariboldi M, et al. Circulating miR-378 in plasma: a reliable, haemolysis-independent biomarker for colorectal cancer. $\mathrm{Br}$ J Cancer. 2014;110(4):1001-7.

15. Hao TB, Shi W, Shen XJ, Qi J, Wu XH, Wu Y, Tang YY, Ju SQ. Circulating cellfree DNA in serum as a biomarker for diagnosis and prognostic prediction of colorectal cancer. Br J Cancer. 2014;111(8):1482-9.

16. Mouliere F, El Messaoudi S, Pang D, Dritschilo A, Thierry AR. Multi-marker analysis of circulating cell-free DNA toward personalized medicine for colorectal cancer. Mol Oncol. 2014;8(5):927-41.

17. Saldova R, Fan Y, Fitzpatrick JM, Watson RW, Rudd PM. Core fucosylation and alpha2-3 sialylation in serum N-glycome is significantly increased in prostate cancer comparing to benign prostate hyperplasia. Glycobiology. 2011;21(2):195-205.

18. Fukushima K, Satoh T, Baba S, Yamashita K. alpha1,2-Fucosylated and beta$\mathrm{N}$-acetylgalactosaminylated prostate-specific antigen as an efficient marker of prostatic cancer. Glycobiology. 2010;20(4):452-60.

19. Robbe-Masselot C, Herrmann A, Maes E, Carlstedt I, Michalski JC, Capon C. Expression of a core 3 disialyl-Le $(x)$ hexasaccharide in human colorectal cancers: a potential marker of malignant transformation in colon. J Proteome Res. 2009:8(2):702-11.

20. Asazawa H, Kamada Y, Takeda Y, Takamatsu S, Shinzaki S, Kim Y, Nezu R, Kuzushita N, Mita E, Kato M, et al. Serum fucosylated haptoglobin in chronic liver diseases as a potential biomarker of hepatocellular carcinoma development. CCLM / FESCC. 2015;53(1):95-102

21. Shiraki K, Takase K, Tameda Y, Hamada M, Kosaka Y, Nakano T. A clinical study of lectin-reactive alpha-fetoprotein as an early indicator of hepatocellular carcinoma in the follow-up of cirrhotic patients. Hepatology. 1995;22(3):802-7.

22. Tanabe K, Deguchi A, Higashi M, Usuki H, Suzuki Y, Uchimura Y, Kuriyama S, Ikenaka K. Outer arm fucosylation of N-glycans increases in sera of hepatocellular carcinoma patients. Biochem Biophys Res Commun. 2008;374(2):219-25.

23. Otake Y, Fujimoto I, Tanaka F, Nakagawa T, Ikeda T, Menon KK, Hase S, Wada $\mathrm{H}$, Ikenaka K. Isolation and characterization of an N-linked oligosaccharide that is significantly increased in sera from patients with non-small cell lung cancer. J Biochem. 2001;129(4):537-42.

24. Park SY, Lee SH, Kawasaki N, Itoh S, Kang K, Hee Ryu S, Hashii N, Kim JM, Kim JY, Hoe Kim J. alpha1-3/4 fucosylation at Asn 241 of beta-haptoglobin is a novel marker for colon cancer: a combinatorial approach for development of glycan biomarkers. Int J Cancer. 2012;130(10):2366-76.

25. Takeda Y, Shinzaki S, Okudo K, Moriwaki K, Murata K, Miyoshi E. Fucosylated haptoglobin is a novel type of cancer biomarker linked to the prognosis after an operation in colorectal cancer. Cancer. 2012;118(12):3036-43.

26. Mikami M, Tanabe K, Matsuo K, Miyazaki Y, Miyazawa M, Hayashi M, Asai S, Ikeda M, Shida M, Hirasawa T, et al. Fully-sialylated alpha-chain of complement 4-binding protein: diagnostic utility for ovarian clear cell carcinoma. Gynecol Oncol. 2015;139(3):520-8.

27. Tanabe K, Kitagawa K, Kojima N, lijima S. Multifucosylated Alpha-1-acid glycoprotein as a novel marker for hepatocellular carcinoma. J Proteome Res. 2016;15(9):2935-44.

28. Bini L, Magi B, Marzocchi B, Cellesi C, Berti B, Raggiaschi R, Rossolini A, Pallin $\checkmark$. Two-dimensional electrophoretic patterns of acute-phase human serum proteins in the course of bacterial and viral diseases. Electrophoresis. 1996; 17(3):612-6.
29. Weivoda S, Andersen JD, Skogen A, Schlievert PM, Fontana D, Schacker T, Tuite P, Dubinsky JM, Jemmerson R. ELISA for human serum leucine-rich alpha-2-glycoprotein-1 employing cytochrome $c$ as the capturing ligand. J Immunol Methods. 2008;336(1):22-9.

30. Norkina O, Kaur S, Ziemer D, De Lisle RC. Inflammation of the cystic fibrosis mouse small intestine. Am J Physiol Gastrointest Liver Physiol. 2004;286(6): G1032-41.

31. Andersen JD, Boylan $\mathrm{KL}$, Jemmerson R, Geller MA, Misemer B, Harrington KM, Weivoda S, Witthuhn BA, Argenta P, Vogel Rl, et al. Leucine-rich alpha2-glycoprotein-1 is upregulated in sera and tumors of ovarian cancer patients. J Ovarian Res. 2010;3:21.

32. Yu KH, Rustgi AK, Blair IA. Characterization of proteins in human pancreatic cancer serum using differential gel electrophoresis and tandem mass spectrometry. J Proteome Res. 2005;4(5):1742-51.

33. Kakisaka T, Kondo T, Okano T, Fujii K, Honda K, Endo M, Tsuchida A, Aoki T, Itoi T, Moriyasu F, et al. Plasma proteomics of pancreatic cancer patients by multi-dimensional liquid chromatography and two-dimensional difference gel electrophoresis (2D-DIGE): up-regulation of leucine-rich alpha-2glycoprotein in pancreatic cancer. J Chromatogr B Anal Technol Biomed Life Sci. 2007:852(1-2):257-67.

34. Okano T, Kondo T, Kakisaka T, Fujii K, Yamada M, Kato H, Nishimura T, Gemma A, Kudoh S, Hirohashi S. Plasma proteomics of lung cancer by a linkage of multi-dimensional liquid chromatography and two-dimensional difference gel electrophoresis. Proteomics. 2006:6(13):3938-48.

35. Wang X, Abraham S, McKenzie JA, Jeffs N, Swire M, Tripathi VB, Luhmann UF, Lange CA, Zhai Z, Arthur HM, et al. LRG1 promotes angiogenesis by modulating endothelial TGF-beta signalling. Nature. 2013;499(7458):306-11.

36. Chachadi VB, Bhat G, Cheng PW. Glycosyltransferases involved in the synthesis of MUC-associated metastasis-promoting selectin ligands. Glycobiology. 2015;25(9):963-75.

37. Sandanayake NS, Sinclair J, Andreola F, Chapman MH, Xue A, Webster GJ, Clarkson A, Gill A, Norton ID, Smith RC, et al. A combination of serum leucine-rich alpha-2-glycoprotein 1, CA19-9 and interleukin-6 differentiate biliary tract cancer from benign biliary strictures. Br J Cancer. 2011;105(9): 1370-8.

38. Fung ET. A recipe for proteomics diagnostic test development: the OVA1 test, from biomarker discovery to FDA clearance. Clin Chem. 2010:56(2):327-9.

\section{Submit your next manuscript to BioMed Central and we will help you at every step:}

- We accept pre-submission inquiries

- Our selector tool helps you to find the most relevant journal

- We provide round the clock customer support

- Convenient online submission

- Thorough peer review

- Inclusion in PubMed and all major indexing services

- Maximum visibility for your research

Submit your manuscript at www.biomedcentral.com/submit
) Biomed Central 